\title{
Remote Imaging Capture with Widefield Swept-Source OCT Angiography During the COVID-19 Pandemic
}

\author{
Itika Garg $\mathbb{D}^{1,2}$, Raviv Katz $\mathbb{D}^{1}$, Yifan Lu $^{1,2}$, John B Miller $\mathbb{D}^{1,2}$ \\ 'Harvard Retinal Imaging Lab, Boston, MA, USA; ${ }^{2}$ Retina Service, Massachusetts Eye and Ear Infirmary, Boston, MA, USA \\ Correspondence: John B Miller, Tel + I 6I7 573-3750, Fax + I 6I7 573-3698, Email john_miller@meei.harvard.edu
}

\begin{abstract}
Purpose: The COVID-19 pandemic has had a widespread impact on the health sector worldwide, both in the clinical and research subsector. This has necessitated for the invention of protocols compliant with social distancing policies to efficiently tackle the current needs. Our aim is to describe a novel remote imaging technique, which helped us continue our research with widefield swept-source optical coherence tomography angiography (WF SS-OCTA).
\end{abstract}

Patients and Methods: As a part of this cross-sectional observational study from August 2020 to September 2020 at Massachusetts Eye and Ear (MEE), we used our institute approved remote desktop, outside of MEE, to gain full access to the on-site WF SS-OCTA device in the imaging room for the purpose of remote imaging. With only the patient being in the imaging room, effective communication was established via video conferencing on an encrypted tablet computer device for the entire procedure.

Results: We imaged four patients with various retinal pathologies, aged 32-69 years. All images obtained were of high quality and signal strength (median score $9 / 10$ for both) and all patients reported a highly satisfactory, comfortable, and safe experience amidst the COVID-19 pandemic.

Conclusion: This novel method is better for remote imaging compared to the previously described techniques as it is time and cost effective, requires limited resources without compromising the image quality or patient satisfaction and adheres to the various guidelines for infection control, most importantly social distancing. As a paradigm shift, this can also be employed in future for setups with limited staff like busy emergency departments or a rural setup with limited access or difficult commute, in the "newnormal" era.

Keywords: COVID-19, COVID, teleophthalmology, remote imaging, teleimaging, telemedicine, optical coherence tomography angiography, retinal imaging, optical coherence tomography

\section{Introduction}

The current coronavirus disease 2019 (COVID-19) pandemic led to a city-wide shut down in Boston, MA, USA starting on March 24th, 2020. ${ }^{1}$ The shut down on research laboratories was ordered even earlier on March 13th, 2020, ${ }^{2}$ with several significant changes in guidelines for both clinical and research practice. Indeed, the Centers for Disease Control and Prevention (CDC) proposed guidelines on ways to mitigate the spread of COVID-19 like social distancing, wearing personal protective equipment, and limiting the occupant capacity of clinics, which have resulted in a paradigm shift in healthcare and research workflow. ${ }^{3,4}$ The social distancing policies vital to the pandemic control have widely impacted the health care sector, ${ }^{5-8}$ in addition to the global economy. Countries or regions with an overwhelmed healthcare system are in desperate need for safe and effective solutions and alternatives to traditional clinical medicine. The impact has been exceptionally challenging to the ophthalmology specialty, ${ }^{9-12}$ which is heavily reliant on diagnostic imaging. In a busy retina clinic, patients potentially interact with many different staff members (ophthalmic technician, photographer, clinician, clinical trainee, etc.) while spending between ten to thirty minutes with each member. Social distancing protocols can be challenging to adhere to during ophthalmic exams and clinical imaging, as the examiner or photographer 
is required to position themselves within a few feet of the patient to operate the device and ensure good image quality. In addition, many patients have preferred telemedicine, telecare, and digital care, if available. ${ }^{13,14}$ This unprecedent crisis with the dire need to protect our health care providers, staff, technicians, and patients have led to invention of new technologies and mainstreaming of existent virtual ophthalmology practices like telemedicine. ${ }^{15}$

While we witnessed a profound influence on patient care across the world, both bench side and clinical research faced an even greater setback. Interestingly, majority of the research laboratories had to shut down except the ones actively engaging in COVID-19 related research. As an example, our Harvard Retinal Imaging Laboratory, actively involved in widefield swept-source optical coherence tomography angiography (WF SS-OCTA) imaging research, withheld all onsite research recruitment from March 14th, 2020, to June 30th, 2020, in compliance with the guidelines. ${ }^{1-4}$ The resumption was slow with many patients hesitant to participate because of the resultant increase in their clinic time duration within close distance of research fellows, even with adequate personal protective equipment and other precautions. Hence, there was a dire need to develop innovative solutions to meet the needs for research imaging, simultaneously ensuring the adequate safety of everyone involved. Previously described methods using an extended High-Definition Multimedia Interface (HDMI) or Digital Visual Interface (DVI) cable ${ }^{16,17}$ for imaging with social distancing (15 to 65 feet) have their inherent drawbacks in the ability to communicate effectively with the patients and requirement of a larger sized room.

Herein, we describe a novel technique for high quality remote research imaging using the latest technological advancements. The photographer employing the remote connection can be both off-site or on-site in a different room, while the patient is alone in the imaging room, maintaining adequate social distancing protocols, for both clinical and research ophthalmology imaging. Thus, this protocol for remote imaging modalities can be potentially utilized universally in ophthalmology clinics and research throughout the COVID-19 pandemic and after.

\section{Materials and Methods}

This cross-sectional observational study was conducted at the Massachusetts Eye and Ear Infirmary (MEEI) from August 2020 to September 2020. All procedures adhered to the tenets of the Declaration of Helsinki and Health Insurance Portability and Accountability Act of 1996. The study was approved by the Institutional Review Board of Massachusetts Eye and Ear Infirmary (2019P001863). A written informed consent was obtained from all patients before being imaged on the WF SS-OCTA device. For this study, patients with the following diagnoses were included as study subjects: age-related macular degeneration, myopia, retinal artery occlusion, and retinal vein occlusion. Exclusion criteria precluded patients aged more than 90 years, inadequate hearing potential, vision $<20 / 200$ and any eyes with glaucoma, dense cataracts, concomitant retinal diseases other than the aforementioned conditions. The patients were imaged on this device after their regular clinical imaging, and hence the patients were pharmacologically dilated during their initial assessment and workup by the ophthalmic technicians, as deemed suitable for the clinical exam.

Figure 1 outlines the schema used for remote imaging. A remote desktop was first verified and approved by the MEEI Research Information Technology (IT) Department to be compliant with the hospital policies and a safe method for using remotely. This device was set up remotely, outside of MEEI, along with a provision of an encrypted tablet computer device (iPad, Apple Inc., Cupertino, CA) to a trained research fellow (I.G.). To ensure compliance with Health Insurance Portability and Accountability Act (HIPAA) guidelines (https://www.hhs.gov/hipaa/index.html), a Network Level Authentication was implemented for the trained research fellow (I.G.) to use the remote desktop for connecting to the onsite workstation at the Harvard Retinal Imaging Laboratory at MEEI. This required the institutional secure username and password for $\log$ in. Further, the research fellow utilized the Microsoft Windows Remote Desktop Connection to operate the $100 \mathrm{kHz}$ WF SS-OCTA instrument (PLEX ${ }^{\circledR}$ Elite 9000, Carl Zeiss Meditec Inc., Dublin, CA) via connecting to its workstation personal computer. To ensure effective communication between the remote research fellow (I.G.) and the patient, another encrypted iPad (Apple Inc., Cupertino, CA) was set up for patients in the imaging room, to allow for clear and effective communication with the fellow via hospital approved video conference calls on hospital network (Zoom Inc, San Jose, California, USA). This enabled real-time, live audio-video synchronous bidirectional communication between the patient and the fellow for the procedure of entire imaging as well as for imaging review, teaching and 


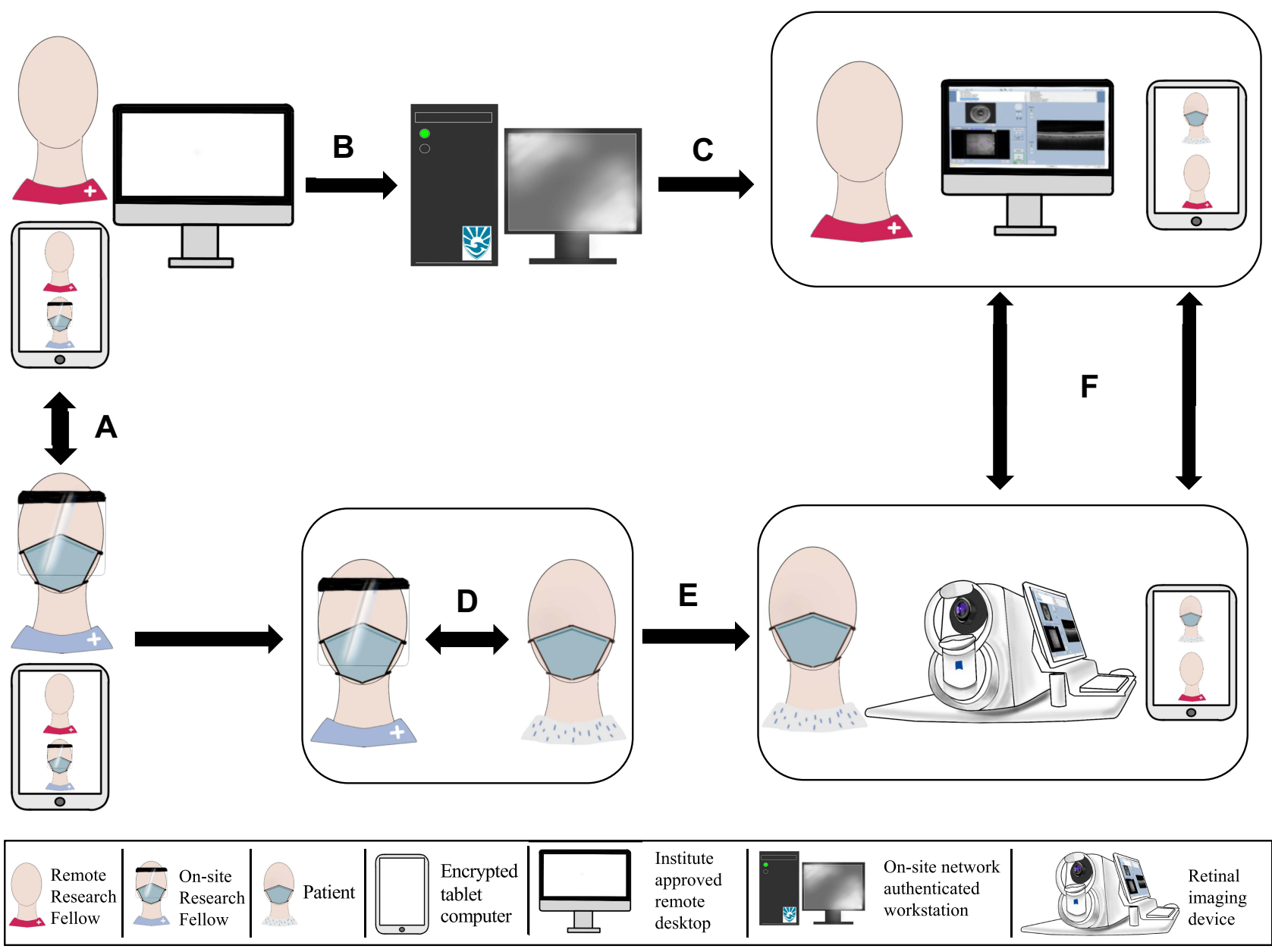

Figure I Workflow schema outlining the novel remote imaging approach used. (A) Both the on-site and off-site fellow communicate with each other as a part of the initial preparation stage. (B) The remote fellow then uses the institution approved remote computer to access an on-site workstation using the institutional user profile, (C) further connecting to and gaining full access to the advanced retinal imaging device. (D) Simultaneously, the on-site research fellow explains the study protocol and obtains a detailed written informed consent, (E) following which they accompany the patient to imaging room with the retinal imaging device and the on-site encrypted tablet computer device. (F) As the last step, the remote fellow uses the remote desktop to navigate the device for imaging and the remote encrypted tablet computer to communicate with the patient.

discussion in the end. At all times, both the on-site workstation and the retinal imaging devices were connected to a power supply.

Ensuring compliance with the CDC guidelines, another on-site research assistant (R.K.) maintained adequate social distancing and hygiene protocols, while obtaining verbal and written informed consent for the research study. Of note, additional explanation during verbal consenting was obtained for patient permission to be imaged by a remote research fellow. The on-site fellow then accompanied the patient to the imaging room introducing them to the power table with the imaging device and encrypted tablet computer with the active Zoom video call with the remote research fellow (Figure 2). Then after the on-site fellow leaves, further instructions were given by the remote fellow regarding their seating position, head rest, chin rest, chin position, and fixation target (green star) to the patient who was alone in the imaging room. The remote fellow used the "acquire" button to reach the acquire screen, following which the pre-decided scan protocol was selected. Further adjustments were made if necessary, using the chinrest controls, live iris viewport, pupil target, live fundus viewport, auto focus, and optimize button; while simultaneously encouraging the patient to make sure his/her head was adequately positioned. Following this, images were obtained along with simultaneous encouragement, instructions, and feedback during the imaging. The patients were instructed to blink normally when necessary and encouraged to close eyes between the various scan protocols to maintain high image quality and avoid artifacts as much as possible. ${ }^{18}$ Adequate patient feedback was also obtained intermittently. In the end, the patients were also explained 


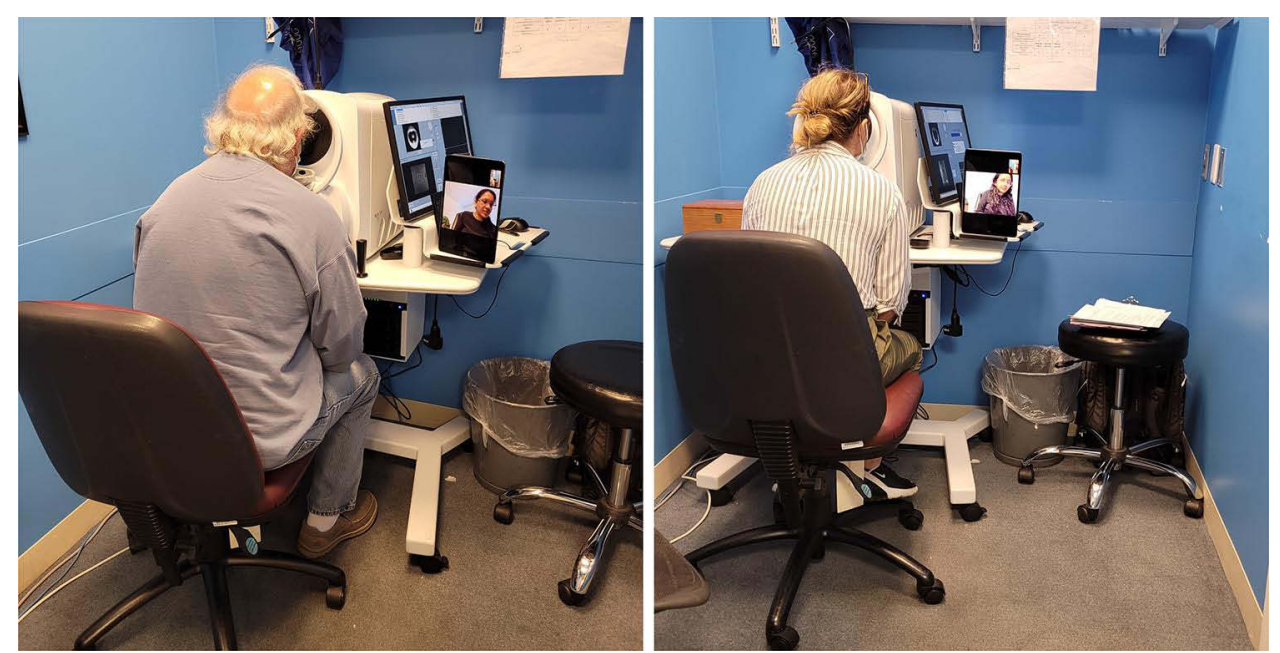

Figure 2 Representative images of patients being remotely imaged using widefield swept-source optical coherence tomography angiography device by an experienced research fellow via an institute approved remote desktop. The relevant communication was done via video conference call on an encrypted tablet device with hospital approved video call application. (Written patient consent obtained for being photographed here for the purpose of publication).

about their findings on the OCTA scan using the "analysis and reports" screen. All the images were obtained and stored on the original retinal imaging device. Of note, in circumstances of shortage of personnel, the same fellow consenting the patient was able to remotely image the patient from the on-site workstation desk (Figure 1C) in a different room on the same floor, hence maintaining safe distance.

\section{Results}

Using the method outlined above, we imaged four patients in the study. Among the four subjects, two patients had a diagnosis of bilateral non-exudative age-related macular degeneration, one patient had a unilateral central retinal vein occlusion, and the last one had a history of previous retinal detachment surgery. The patients' age ranged from 32 years old to 69 years old, and $50 \%$ of them were females (Table 1). Both eyes of all patients were included in the study.

For each subject, Angio $3 \mathrm{~mm} \times 3 \mathrm{~mm}, 6 \mathrm{~mm} \times 6 \mathrm{~mm}$, and $12 \mathrm{~mm} \times 12 \mathrm{~mm}$ OCT angiograms were obtained (Figure 3). The quality of images taken remotely was measured by the automatic scoring system of the device, displayed on the quality check screen after completion of imaging. All images obtained surpassed the minimum image quality score required for our previous publications (7/10) with the median quality score being $9 / 10$. The image quality score ranged from a minimum of 8/10 to a maximum of 10/10. All images qualified for inclusion in the study. Scan length, depth, signal strength, and duration were also included as indicators for image quality, provided in Table 1 . The scan duration ranged from 7.2 seconds to 58 seconds (mean 19.7 seconds, median 13.2 seconds).

All patients reported no difficulty in comprehension of the consenting procedures as well as the instructions received via video conferencing, despite being alone in the room during the entire acquisition procedure. All patients reported excellent user feedback with the experience and welcomed the effort of the research team to ensure their safety and effectively implementing the social distancing policy in an otherwise small room which can be an unnecessary source of anxiety and discomfort. All patients opted in for detailed explanations of their OCTA scans and voiced understanding of the use of the screen cursor highlighting the regions of interest, being controlled remotely. All patients reported their experience to be worth their time and effort, along with feeling safe amidst a pandemic.

\section{Discussion}

The COVID-19 pandemic has impacted the world tremendously, inducing drastic changes in the practice of clinical medicine, research, and education. The guidelines set forth by the CDC has simultaneously imposed a significant challenge to the field of ophthalmology because of heavy reliance on diagnostic imaging being an essential component of daily clinical practice, research and education. ${ }^{15,19-22}$ We describe a safe and efficient novel technique for high quality 
Table I Demographic Characteristics of Patients Imaged Remotely, Along with the Resultant Image Quality Metrics

\begin{tabular}{|c|c|c|c|c|c|c|c|c|}
\hline Patient Number & Age (Years) & Sex & Eye & Scan Protocol & Scan Length (mm) & Scan Depth (mm) & Signal Strength & $\begin{array}{c}\text { Scan Duration } \\
\text { (Seconds) }\end{array}$ \\
\hline \multirow[t]{7}{*}{ I } & \multirow[t]{7}{*}{69} & \multirow[t]{7}{*}{ Female } & \multirow[t]{3}{*}{ OD } & Angio $(3 \mathrm{~mm} \times 3 \mathrm{~mm})$ & 3 & 3 & 9 & 8.1 \\
\hline & & & & Angio $(6 \mathrm{~mm} \times 6 \mathrm{~mm})$ & 6 & 3 & 10 & 11.6 \\
\hline & & & & Angio $(12 \mathrm{~mm} \times 12 \mathrm{~mm})$ & 12 & 3 & 9 & 18.4 \\
\hline & & & \multirow[t]{4}{*}{ OS } & Angio $(3 \mathrm{~mm} \times 3 \mathrm{~mm})$ & 3 & 3 & 9 & 11.5 \\
\hline & & & & Angio $(6 \mathrm{~mm} \times 6 \mathrm{~mm})$ & 6 & 3 & 10 & 14.3 \\
\hline & & & & Angio (12 mm x $12 \mathrm{~mm})$ & 12 & 3 & 9 & 42.4 \\
\hline & & & & HD Angio $(12 \mathrm{~mm} \times 12 \mathrm{~mm})$ & 12 & 6 & 8 & 39.7 \\
\hline \multirow[t]{10}{*}{2} & \multirow[t]{10}{*}{67} & \multirow[t]{10}{*}{ Male } & \multirow[t]{5}{*}{ OD } & Angio $(3 \mathrm{~mm} \times 3 \mathrm{~mm})$ & 3 & 3 & 8 & 7.2 \\
\hline & & & & Angio $(6 \mathrm{~mm} \times 6 \mathrm{~mm})$ & 6 & 3 & 9 & 9.4 \\
\hline & & & & Angio $(12 \mathrm{~mm} \times 12 \mathrm{~mm})$ & 12 & 3 & 8 & 9.3 \\
\hline & & & & Angio $(12 \mathrm{~mm} \times 12 \mathrm{~mm})$ & 12 & 3 & 8 & 12.2 \\
\hline & & & & Angio $(6 \mathrm{~mm} \times 6 \mathrm{~mm})$ & 6 & 3 & 9 & 11.9 \\
\hline & & & \multirow[t]{5}{*}{ OS } & Angio $(3 \mathrm{~mm} \times 3 \mathrm{~mm})$ & 3 & 3 & 9 & 8.8 \\
\hline & & & & Angio $(6 \mathrm{~mm} \times 6 \mathrm{~mm})$ & 6 & 3 & 10 & 10.2 \\
\hline & & & & Angio $(6 \mathrm{~mm} \times 6 \mathrm{~mm})$ & 6 & 3 & 10 & 11.5 \\
\hline & & & & Angio $(12 \mathrm{~mm} \times 12 \mathrm{~mm})$ & 12 & 3 & 9 & 17.8 \\
\hline & & & & Angio $(12 \mathrm{~mm} \times 12 \mathrm{~mm})$ & 12 & 3 & 9 & 22.2 \\
\hline
\end{tabular}


Table I (Continued).

\begin{tabular}{|c|c|c|c|c|c|c|c|c|}
\hline Patient Number & Age (Years) & Sex & Eye & Scan Protocol & Scan Length $(\mathrm{mm})$ & Scan Depth (mm) & Signal Strength & $\begin{array}{l}\text { Scan Duration } \\
\text { (Seconds) }\end{array}$ \\
\hline \multirow[t]{6}{*}{3} & \multirow[t]{6}{*}{32} & \multirow[t]{6}{*}{ Female } & \multirow[t]{3}{*}{ OD } & Angio $(3 \mathrm{~mm} \times 3 \mathrm{~mm})$ & 3 & 3 & 9 & 25.3 \\
\hline & & & & Angio $(6 \mathrm{~mm} \times 6 \mathrm{~mm})$ & 6 & 3 & 10 & 12.6 \\
\hline & & & & Angio $(12 \mathrm{~mm} \times 12 \mathrm{~mm})$ & 12 & 3 & 9 & 26.2 \\
\hline & & & \multirow{3}{*}{ os } & Angio $(6 \mathrm{~mm} \times 6 \mathrm{~mm})$ & 6 & 3 & 10 & 13.8 \\
\hline & & & & Angio $(12 \mathrm{~mm} \times 12 \mathrm{~mm})$ & 12 & 3 & 9 & 34.4 \\
\hline & & & & Angio $(12 \mathrm{~mm} \times 12 \mathrm{~mm})$ & 12 & 3 & 9 & 56.3 \\
\hline \multirow[t]{3}{*}{4} & \multirow[t]{3}{*}{66} & \multirow[t]{3}{*}{ Male } & OD & Angio $(3 \mathrm{~mm} \times 3 \mathrm{~mm})$ & 3 & 3 & 9 & 9.1 \\
\hline & & & \multirow{2}{*}{ OS } & Angio $(6 \mathrm{~mm} \times 6 \mathrm{~mm})$ & 6 & 3 & 10 & 51.0 \\
\hline & & & & Angio $(12 \mathrm{~mm} \times 12 \mathrm{~mm})$ & 12 & 3 & 9 & 58.0 \\
\hline Mean & & & & & & & 9.25 & 19.7 \\
\hline Median & & & & & & & 9 & 13.2 \\
\hline
\end{tabular}



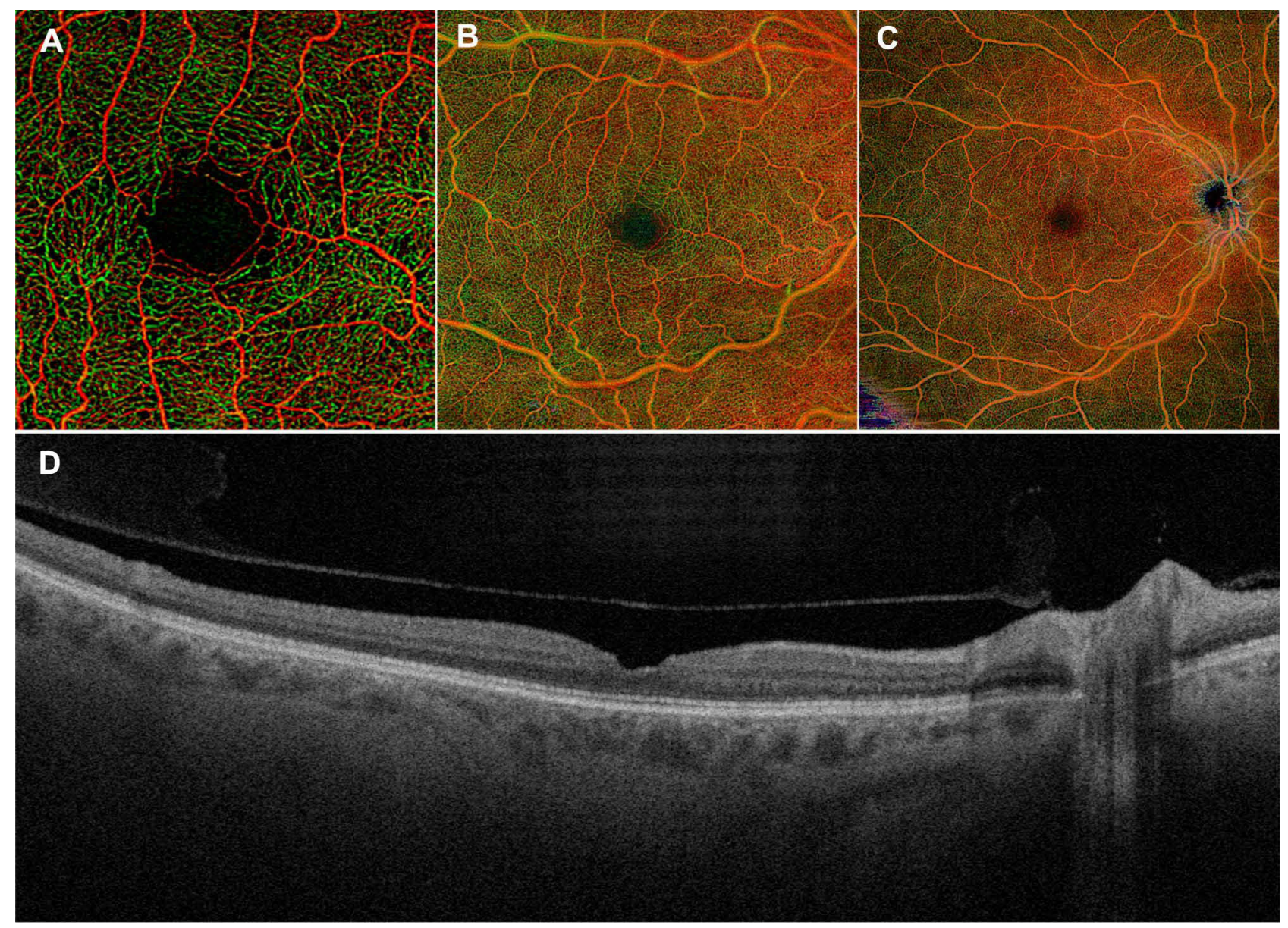

Figure 3 Representative Angio $3 \mathrm{~mm} \times 3 \mathrm{~mm}$ (A), Angio $6 \mathrm{~mm} \times 6 \mathrm{~mm}$ (B), $12 \mathrm{~mm} \times 12 \mathrm{~mm}$ scans (C), and the Cube $800 \times 800$ B-scan (D) from the right eye of patient number I in Table I, obtained via the remote imaging technique outlined.

remote imaging which can allow both the continuation of imaging research as well as clinical imaging during the pandemic ensuring social distancing protocols. This technique can serve as a foundation for telemedicine based digital imaging that can be used in future for situations with limited staffing in a busy clinic setting like an emergency department, or in a distant rural area setup inaccessible or not feasible by an easy commute. This can also be employed for training and education purposes remotely with new photographers while they are in the imaging room with the patient. Recent technological improvements like tremendously improved bandwidth of modern internet services, hospital based virtual private network services, improved video conference call quality, and advanced computer gadgets have played a key role in patient acceptance, provider comfort and supreme imaging quality. Further, it has been shown that using a tablet computer device with larger screens over a mobile device results in better visualization and thereby quicker patient acceptance. $^{15}$

In recent times, various groups have explored the realm of remote imaging. Zeiss Meditec, Inc. (Jena, Germany) developed a method to remotely image patients using an extended HDMI or DVI cable that allows photographers to control the device from approximately fifteen feet away. ${ }^{16,17}$ This method provides photographers with the option to image patients while adhering to social distancing guidelines. This technique, however, makes it difficult for photographers and patients to communicate and requires additional space. Zeiss Meditec, Inc. also employs an additional method to remotely image patients using the spectral domain Cirrus device with TeamViewer (TeamViewer AG, Germany). This allows photographers and patients to communicate via video conferencing. However, this method requires a license for operating TeamViewer in conjunction with the machine by our institution, thus contributing to an additional financial burden to already existing research protocols. 
Recently, there have been studies highlighting the emerging applications of portable and handheld OCT systems in non-traditional settings. ${ }^{23-25}$ Mehta et al have utilized a web-based user interface being accessed on a cellular phone mounted to a handheld OCT probe, ${ }^{26}$ for the wireless control of the imaging device, ${ }^{27}$ however, it does not meet the needs of social distancing between the photographer having the OCT probe and the patient. However, with this software, multiple users can review and control the same imaging session remotely in another remote via the same web-based user interface on various devices like cellular phone, tablets, or laptop. Another new device, Notal Vision (Tel Aviv, Israel) Home OCT, allows at-home OCT imaging with direct transmission of data to the ophthalmologist; however, it is not approved for reimbursement through insurance companies. ${ }^{28}$ Thus, identifying the most effective method to remotely image patients during the COVID-19 pandemic has remained a challenge, especially with HIPAA and security concerns. A benefit of our approach is its cost-effectiveness, as purchasing an additional license or program was not required. As discussed in our prior work on telehealth implementation, we used Zoom approved for healthcare use because of its improved privacy (ie, multilayer security with AES-256 encryption) and its ability to integrate with electronic health records. ${ }^{19}$ Most importantly, our approach of imaging which included a remote and an on-site fellow, helped us adhere to the CDC guidelines of adequate hygiene, sanitization, use of personal protective equipment along with social distancing, and thereby minimize the risk of spread of the COVID-19 virus. Our approach made the patients feel safe and comfortable with high satisfaction ratings as they are alone in the imaging room.

Despite promising results, our study also has certain notable limitations. First, the sample size is comparatively smaller, because of the pandemic related decline in the overall number of hospital visits. ${ }^{11}$ Additionally, it was difficult to motivate and recruit patients for their participation in clinical research as the overall goal was to keep the exposure time in clinic setting to a minimum. This is especially relevant for the age group of patients being imaged, primarily having a diagnosis of age-related macular degeneration. Despite this, the acquired images were consistently shown to be highquality as a part of our ongoing prospective study. Also, Zeiss PLEX ${ }^{\circledR}$ Elite 9000 WF SS-OCTA was the only device assessed for this technique. Compared to other OCTA devices such as SPECTRALIS ${ }^{\circledR}$ (Heidelberg Engineering, Heidelberg, Germany) and DRI OCT Triton (Topcon corporation, Tokyo, Japan), the PLEX ${ }^{\circledR}$ Elite 9000 WF SSOCTA is potentially more suitable for remote imaging as minor chin adjustments are sufficient to maintain focus while performing imaging. Other machines require manual manipulation of the joystick during the imaging sessions, thereby presenting an additional obstacle to the development of remote imaging techniques. However, this study with the PLEX $^{\circledR}$ Elite 9000 WF SS-OCTA may serve as an example to illustrate the high-quality remote imaging on OCTA devices that can be achieved. With this study, we provide a potential foundation for remote imaging on all other OCTA devices in the future.

In conclusion, our novel approach for high quality remote imaging not only enables the scientific community to continue the research, but also allows physicians to continue their diagnostic imaging in Ophthalmology. It can also be used to prevent the subsequent resurgence of infection with current gradual reopening and relaxation of the CDC guidelines. Our experience at the time of writing this shows extreme patient satisfaction and good quality imaging, which has helped us continue our research in a completely redefined fashion during the pandemic, while ensuring safety for all. We have also employed this method for remotely imaging through a device in our 24-hours emergency department with similar results. Hence, we hope more providers and institutes will embrace these changes and advancements which are the need of hour, stimulating innovations to transform health care delivery and efficiently adapt to the new-normal. This could also mean reliable and efficient home testing models, along with a possibility of self-monitoring on smartphones via the application of artificial intelligence. While we await such models to develop and be widely available, these current efforts are likely to have strong impact in making teleophthalmology and telemedicine based digital imaging more mainstream.

\section{Ethics Approval and Informed Consent}

All procedures adhered to the tenets of the Declaration of Helsinki and Health Insurance Portability and Accountability Act of 1996. The study was approved by the Institutional Review Board of Massachusetts Eye and Ear Infirmary (2019P001863). A written informed consent was obtained from all patients for being imaged on the device. 


\section{Consent for Publication}

Written patient consents were obtained for being photographed for the purpose of publication.

\section{Acknowledgments}

The authors would like to thank all the staff for their help and assistance in research at retina service, MEEI.

\section{Author Contributions}

All authors made a significant contribution to the work reported, whether that is in the conception, study design, execution, acquisition of data, analysis, and interpretation, or in all these areas; took part in drafting, revising or critically reviewing the article; gave final approval of the version to be published; have agreed on the journal to which the article has been submitted; and agree to be accountable for all aspects of the work.

\section{Funding}

This research was funded by Lions International Grant, grant number 530125 and 530869. The funding organization had no role in the study design or conduct of this research.

\section{Disclosure}

I.G., R.K. and Y.L. report no conflicts of interest in this work. J.B.M. is a consultant to Carl Zeiss, Alcon, Allergan, Sunovion, Topcon and Genentech.

\section{References}

1. Governor Charlie Baker orders all non-essential businesses to cease in person operation, directs the department of public health to issue stay at home advisory for two weeks; 2020. Available from: https:/www.mass.gov/news/governor-charlie-baker-orders-all-non-essential-businesses-tocease-in-person-operation. Accessed February 18, 2022.

2. Coronavirus: the first three months as it happened. Nature; 2020. Available from: https://www.nature.com/articles/d41586-020-00154-w. Accessed February 18, 2022.

3. Centers for Disease Control and Prevention. Get your mass gatherings or large community events ready for coronavirus disease $2019 ; 2020$. Available from: https://www.cdc.gov/coronavirus/2019-ncov/community/large-events/mass-gatherings-ready-for-covid-19.html. Accessed February $18,2022$.

4. Centers for Disease Control and Prevention. Interim guidance for implementing safety practices for critical infrastructure workers who may have had exposure to a person with suspected or confirmed COVID-19; 2020. Available from: https://www.cdc.gov/coronavirus/2019-ncov/community/ critical-workers/implementing-safety-practices.html. Accessed February 18, 2022.

5. Garcia S, Albaghdadi MS, Meraj PM, et al. Reduction in ST-segment elevation cardiac catheterization laboratory activations in the United States during COVID-19 pandemic. J Am Coll Cardiol. 2020;75(22):2871-2872. doi:10.1016/j.jacc.2020.04.011

6. Tam CCF, Cheung KS, Lam S, et al. Impact of coronavirus disease 2019 (COVID-19) outbreak on ST-segment-elevation myocardial infarction care in Hong Kong, China. Circ Cardiovasc Qual Outcomes. 2020;2019:2019-2021. doi:10.1161/CIRCOUTCOMES.120.006631

7. Metzler B, Siostrzonek P, Binder RK, Bauer A, Reinstadler SJ. Decline of acute coronary syndrome admissions in Austria since the outbreak of COVID-19: the pandemic response causes cardiac collateral damage. Eur Heart J. 2020;41(19):1852-1853. doi:10.1093/eurheartj/ehaa314

8. Schirmer CM, Ringer AJ, Arthur AS, et al. Delayed presentation of acute ischemic strokes during the COVID-19 crisis. J Neurointerv Surg. 2020;12(7):639-642. doi:10.1136/neurintsurg-2020-016299

9. Wickham L, Hay G, Hamilton R, et al. The impact of COVID policies on acute ophthalmology services — experiences from Moorfields eye hospital NHS foundation trust. Eye. 2020;34(7):1189-1192. doi:10.1038/s41433-020-0957-2

10. Stone LG, Grinton ME, Talks JS. Delayed follow-up of medical retina patients due to COVID-19: impact on disease activity and visual acuity. Graefe's Arch Clin Exp Ophthalmol. 2021;259(7):1773-1780. doi:10.1007/s00417-021-05174-4

11. Moon JY, Miller JB, Katz R, et al. The impact of the COVID-19 pandemic on ophthalmic care at an eye-specific emergency department in an outbreak hotspot. Clin Ophthalmol. 2020;14:4155-4163. doi:10.2147/OPTH.S285223

12. Patel LG, Peck T, Starr MR, et al. Clinical presentation of rhegmatogenous retinal detachment during the COVID-19 pandemic: a historical cohort study. Ophthalmology. 2021;128(5):686-692. doi:10.1016/j.ophtha.2020.10.009

13. Predmore ZS, Roth E, Breslau J, Fischer SH, Uscher-Pines L. Assessment of patient preferences for telehealth in post-COVID-19 pandemic health care. JAMA Netw Open. 2021;4(12):e2136405. doi:10.1001/jamanetworkopen.2021.36405

14. Drerup B, Espenschied J, Wiedemer J, Hamilton L. Reduced no-show rates and sustained patient satisfaction of telehealth during the COVID-19 pandemic. Telemed e-Health. 2021;27(12):1409-1415. doi:10.1089/tmj.2021.0002

15. Saleem SM, Pasquale LR, Sidoti PA, Tsai JC. Virtual ophthalmology: telemedicine in a COVID-19 era. Am J Ophthalmol. 2020;216:237-242. doi:10.1016/j.ajo.2020.04.029

16. Practice social distancing with your patient exams using ZEISS CIRRUS; 2021. Available from: https://www.zeiss.com/content/dam/med/ref international/corona/pdfs/zeiss_social_distance_cirrus_cableconnection.pdf. Accessed February 18, 2022. 
17. Quickstart Guide. Social distancing with your ZEISS CIRRUS extended cable connection; 2021. Available from: https://www.zeiss.com/content/ dam/med/ref_international/corona/pdfs/cirrus-cable-connection-quickstart.pdf. Accessed February 18, 2022.

18. Cui Y, Zhu Y, Wang JC, et al. Imaging artifacts and segmentation errors with wide-field swept-source optical coherence tomography angiography in diabetic retinopathy. Transl Vis Sci Technol. 2019;8:6. doi:10.1167/tvst.8.6.18

19. Lu ES, Houston SKS, Rahimy E, Miller JB. Telehealth implementation in retina practices during COVID-19. Retin Today. 2020;2020:46-49.

20. Lu ES, Miller JB, Houston SKS, Kitchens JW. Live-streaming from the or using a plug-and-play device. Retin Today. 2020;2020:47-49.

21. Park CH, Rahimy E, Shahlaee A, Federman JL. Retina today: telemedicine in ophthalmology. Retin Today. 2017;2017:55-58.

22. Lu ES, Reppucci VS, Houston SKS. Three-dimensional telesurgery and remote proctoring over a 5G network. Digit J Ophthalmol. 2021;27 (3):38-43. doi:10.5693/djo.01.2021.06.003

23. Rufai SR. Handheld optical coherence tomography removes barriers to imaging the eyes of young children. Eye. 2022. doi:10.1038/s41433-02101884-5

24. Chopra R, Wagner SK, Keane PA. Optical coherence tomography in the 2020s—outside the eye clinic. Eye. 2021;35(1):236-243. doi:10.1038/ s41433-020-01263-6

25. Ni S, Wei X, Ng R, et al. High-speed and widefield handheld swept-source OCT angiography with a VCSEL light source. Biomed Opt Express. 2021;12(6):3553. doi:10.1364/boe.425411

26. Nankivil D, Waterman G, LaRocca F, Keller B, Kuo AN, Izatt JA. Handheld, rapidly switchable, anterior/posterior segment swept source optical coherence tomography probe. Biomed Opt Express. 2015;6(11):4516. doi:10.1364/boe.6.004516

27. Mehta R, Nankivil D, Zielinski DJ, et al. Wireless, web-based interactive control of optical coherence tomography with mobile devices. Transl Vis Sci Technol. 2017;6(1):5. doi:10.1167/tvst.6.1.5

28. Maloca P, Hasler PW, Barthelmes D, et al. Safety and feasibility of a novel sparse optical coherence tomography device for patient-delivered retina home monitoring. Transl Vis Sci Technol. 2018;7(4):8. doi:10.1167/tvst.7.4.8

Clinical Ophthalmology

\section{Dovepress}

\section{Publish your work in this journal}

Clinical Ophthalmology is an international, peer-reviewed journal covering all subspecialties within ophthalmology. Key topics include: Optometry; Visual science; Pharmacology and drug therapy in eye diseases; Basic Sciences; Primary and Secondary eye care; Patient Safety and Quality of Care Improvements. This journal is indexed on PubMed Central and CAS, and is the official journal of The Society of Clinical Ophthalmology (SCO). The manuscript management system is completely online and includes a very quick and fair peer-review system, which is all easy to use. Visit http://www. dovepress.com/testimonials.php to read real quotes from published authors.

Submit your manuscript here: https://www.dovepress.com/clinical-ophthalmology-journal 